\title{
MUSIKANALYSE UND MUSIKVERMITTLUNG
}

\author{
Hans Heinrich Eg g e b r e cht (Freiburg i. Br.)
}

Musikanalyse, wörtlich: die 'Auflösung' eines musikalisch Gegebenen in seine Bestandteile, eines musikalischen Sinngefüges in seine Sinnträger, und dem Sinne nach: die Erkundung der Intentions-, Daseins- und Wirkungsstruktur eines solcher Erkundung sich anbietenden, sie herausfordernden musikalisch konkreten Gefüges, ist ein Teil der auf Musik gerichteten Wissenschaft. Und in dem Maße, in dem in der Geschichte der Musik das musikalische Konkretum, die Komposition, das Werk, an Charakter, Individualität, Durchdachtheit, Einmaligkeit gewann und dies besonders seit der Wiener Klassik und dann speziell seit der Wiener Schönberg-Schule und ihrer allgemeinen Aufwertung und Ausstrahlung nach 1950, rückte innerhalb der Geschichte der Musikwissenschaft die musikalische Analyse in den Vordergrund, wurde - in Ergänzung oder Ablösung der Stilforschung - zur Basis aller konkret auf Musik gerichteten wissenschaftlichen Arbeit, problematisierte und verfeinerte sich methodologisch und begann - auch im Griff nach den Möglichkeiten der Semiotik, Informationstheorie und Computertechnik - sich auszubreiten weit über die artifizielle, professionell komponierte, der Kunst zugehörige Musik hinaus:

Musikanalyse als Erkundung dessen, was das musikalische Ding an und für sich eigentlich sei, wie es gemacht und gemeint ist, behauptet sich als der heutigen Musikwissenschaft wichtigster Teil. Und dies gemäß der Maxime, daß Aussagen über Musik - auch dort, wo sie philosophisch nach deren Wesen fragen, - und daß Urteile über den ästhetischen Wert, die historische Position, die soziale Funktion und Wirkungsform musikalischer Phänomene nur dann Verbindlichkeit und Überzeugungskraft gewinnen können, wenn sie - analytisch - an der Musik selbst festgemacht worden sind. Und dies wird so bleiben, - nicht vorstellbar, daß es anders wird, solange es Musik gibt im Sinne eines Begriffs von Kunst, bei dem das erdachte Gebilde einen Sinn hat, den es - intentional ästhetisch - durch sich selbst stiftet und mitteilt. 
Daß die als Wissenschaft sich verstehende Musikanalyse methodologische Probleme zeigt, an Grenzen ihrer Reichweite stößt und in der heute verbreiteten analytischen Geschäftigkeit negativen Tendenzen ausgeliefert sein kann, das hat sie mit anderen musikwissenschaftlichen Arbeitsbereichen gemeinsam.

Eines der methodologischen Probleme sehe ich nach wie vor in dem Wissenschaftsanspruch musikalischer Analyse als gleichwohl und unabwendbar subjektiv interpretatorischer Leistung; diesem Problem begegne ich persönlich mit dem ausdrücklichen Bekenntnis zur Subjektivität des analytischen Zugriffs, der das Subjekt intentional zu einer Instanz erhebt, ohne die das Ding der Kunst nicht sein kann. Ein anderes Problem sehe ich in dem Widerstreit zwischen der Geschichtsbedingtheit und dem bleibenden Wert des zu analysierenden Objekts; diesem Problem begegne ich mit der gleichsam wissenschaftlichen Anerkennung der ästhetischen Faszination, die die zu analysierende Musik auf mich ausübt und in der ihre Geschichtsbedingtheit, die sie vorzeigt, zugleich zur Gegenwart hin aufgehoben, getilgt ist. Ein drittes Problem sehe ich (immer wieder) in der Frage der konkreten gesellschaftlichen Ableitung eines als Kunst konzipierten ästhetischen Gebildes; ihr begegne ich mit dem Bewußtsein der wenn auch gesellschaftlich determinierten, so doch inmitten aller Determination potentiellen Freiheit des Ichs, dem die Determination und zugleich Freiheit der ästhetischen Kreation einen Spiegel vorhält. Ein wiederum anderes Problem sehe ich (immer noch) in der Notwendigkeit und zugleich Schwierigkeit der stringenten Ausweitung der traditionellen Formanalyse um die Dimension des Gehalts; hier aber leitet mich die Überzeugung, daß nichts und am wenigsten Musik nur Form sein kann, und der immer wieder erneute Versuch, auch dasjenige analytisch zur Wissenschaft $\mathrm{zu}$ bringen, was bei Musik mehr ist als Form.

Die Grenzen der Reichweite der Analyse von Musik liegen in ihrer Daseinsart beschlossen, die dadurch gekennzeichnet ist, daß sie das sprachlos existierende, begriffslos wirkende ästhetische Sinngebilde zur Sprache bringt, zum Begriff. Im Erkennen und Benennen sinnlich gestifteten Sinns (dem die Gehalte innewohnen), in der Transformation solcher Sinnstiftungen zur begrifflichen Bewußtheit besteht ihre Leistung, wobei sie an das der Musik inhärente traditionelle und innovationelle begriffliche Denken anknüpft, es zum Einzelfall hin konkretisiert, differenziert und bereichert, um dadurch die Musik zur Wissenschaft $\mathrm{zu}$ bringen und zugleich womöglich dem spezifisch ästhetischen (sinnlichen, begriffslosen) Verstehen hilfreich zu sein, das nach solcher Hilfe verlangt.

Indessen hat die Musikanalyse ihre Begrenztheit gegenüber ihrem Gegenstand, der Musik, ebenso selbstverständlich wie grundsätzlich zunächst darin, daß in ihrem Medium, dem der Sprache, das ästhetische Ding selbst und als solches nicht mehr existent ist. Auch kann sie nur dessen Sinngehaltigkeit erklären und beweisen, nicht aber seine Schön- 
heit. Und selbst bei einem einfachen musikalischen Sinngebilde, einem Thema zum Beispiel oder einer Klangfortschreitung, kann sie auf ihrer Ebene der Sinnerklärung die Vielschichtigkeit, Komplexität und Interdependenz der ästhetischen Definitionsprozesse des Sinns niemals erschöpfend erfassen, während sie den Gehalt von Musik überhaupt nur in der Weise von Begriffsfeldern, Metaphern und Gleichnissen zur Sprache $\mathrm{zu}$ bringen vermag.

Darüber hinaus aber sollte die musikalische Analyse, indem sie ihre Arbeit verrichtet, von dem Bewußtsein durchdrungen bleiben, daß, wie alle Kunst, so auch die Musik, sie jedoch am ausgeprägtesten, zwei ineinander verwobene Bereiche hat, einen emotionalen, gefühlsmäßigen, unmittelbar der Seele zugekehrten und einen rationalen, der dem Verstande zugänglich ist, und daß das Spezifische der Musik, ihre Sinnenhaftigkeit, in der das Kunstschöne wohnt, in dem erstgenannten Bereich gelegen ist. Musikalische Analyse als Transformation musikalischen Sinns und Gehalts in die Sphäre des Begriffs kann am Phänomen der Musik, ihrem Gegenstand, nur dasjenige zur Sprache bringen, was sprachlich erfaßbar ist, d. h. nur das Rationale und Rationalisierbare der Musik, dasjenige an ihr, was dem Verstande zugewandt ist als dem Vermögen des Denkens in Begriffen. Das analytisch nicht Faßbare, nicht Sagbare ist hier nicht der als das Irrationale übrigbleibende Rest, sondern ist das spezifisch Ästhetische, in dessen Reich der Bereich des Sagbaren zwar konstitutiv und doch zugleich aufgehoben ist ins Ganze eines der Seele zugekehrten Doppelbereichs.

Negative Tendenzen heutiger Musikanalyse sehe ich in deren geschäftiger Veräußerlichung, die sich unter anderem darin anzeigt, daß die Analyse im bloßen Beschreiben von Musik steckenbleibt, daß sie unreflektiert formalistisch verfährt und - was das schlimmste ist daß sie keine je besonderen Aufgaben sich stellt, nicht in übergeordnete Fragen sich einbindet, die es analytisch $\mathrm{zu}$ beantworten gilt. Negativ darüber hinaus muß es sich auswirken, wenn Musikanalyse in bezug auf ihre Problematik und ihre Grenzen zu selbstverständlich sich gebärdet und $\mathrm{zu}$ fragen vergißt, wozu überhaupt sie da ist, - eine Frage, die jedesmal wenn nicht neu gestellt, so doch mitbedacht werden sollte, um dem Irrtum zu begegnen, der die Musik zergliedernde und auflösende Zugriff sei schon wertvoll, wenn überhaupt er geschieht.

Schwer jedoch und schwerer als die offensichtlichen Analyseschwächen, die - etwa im Unterricht - nicht selten durch einen einfachen Fingerzeig und zumeist durch Gegenbeispiele bewußt gemacht werden können, wiegt die Tendenz musikanalytischer Arbeit zu ihrer Verselbständigung in dem Sinne, daß sie den Bezug zu der spezifisch ästhetischen Entstehungs-, Daseins- und Wirkungsweise ihres Gegenstandes einbüßt, indem sie ihn im Eifer des Sehens, Denkens und Versprachlichens buchstäblich aus den Ohren verliert und ins papierene Getüftel ableitet. 
Dem antiästhetischen Gebaren solcher Verselbständigung benachbart und zugleich ein Spiegel der allgemeinen Verkümmerung der Empfindungskräfte ist der heute verbreitete Glaube an die Allmacht der musikalischen Analyse, der so tut, als sei das Rationale, das sprachlich Erfaßbare der Musik deren Hauptsache und das Emotionale nur eben jener irrationale Rest, über den nicht weiter zu handeln sei. Was der Analyse heute nottut, im Unterricht der Schule wie der Hochschule, im Rahmen der Aktivitäten, durch die die Musik zur Wissenschaft gebracht wird, ebenso wie im Dienste der Förderung des Musikverstehens, ist die Zurechtrückung des Verhältnisses zwischen Ratio und Sensus in die Richtung, daß beide zusammen das musikalische Ding konstituieren, dessen Eigentümlichkeit jedoch darin besteht, daß es in beidem unmittelbar zur Empfindung ist. Musikalische Analyse als der nach wie vor zentrale Teilbereich heutiger Musikwissenschaft sollte nicht nur die intellektuelle Potenz gegenüber dem ästhetischen Gegenstand entwickeln, sondern zugleich die ästhetische Potenz vor der Gefahr ihrer intellektuellen Erstickung bewahren.

Wie nun dies $\mathrm{zu}$ geschehen hat: nicht nur die (eigentlich selbstverständliche) beständige Kontrolle der analytischen Aussagen seitens der erklingenden Musik, sondern auch die zugleich mit dem sprachlichen Zugriff erfolgende bewußte Einbeziehung, die geplante Einkalkulierung des Unaussprechbaren, die Thematisierung der Begrenztheit der Sprache gegenüber dem Spezifischen der Musik, dem Sinnlichen und Emotionalen als der eigentlichen Instanz des ästhetischen Gebildes, wie dies im Rahmen der wissenschaftlichen musikalischen Analyse konkret geschehen könnte, soll hier nicht weiter ausgeführt werden. Vielmehr ist es die Intention dieses Textes, hieran lediglich erinnert $\mathrm{zu}$ haben, um in diesem Zusammenhang auf eine Unzulänglichkeit der musikalischen Analyse hinzuweisen, die auch dort bestehen kann und weitesthin besteht, wo sie als Wissenschaft ihre negativen Tendenzen vermeidet, ihre Probleme reflektiert und die Grenzen ihrer Reichweite einsieht, mit einem Wort: wo sie in ihren Möglichkeiten als wissenschaftlich gelungen anzusehen ist. Das Unzulängliche, der Mangel, das Unzureichende der sieh als Wissenschaft verstehenden Musikanalyse gründet in ihrem Selbstverständnis als Wissenschaft, das an und für sich zwar legitim und im Wissenschaftsbereich unverzichtbar ist, jedoch versagt, sobald die wissenschaftliche Analyse von Musik vor ein Publikum tritt, der Analytiker sich Menschen gegenübergestellt sieht, denen er auf sprachlichem Wege beim Verstehen von Musik helfen soll oder möchte.

Wissenschaft nämlich, so auch die im Zeichen von Wissenschaft unternommene Musikanalyse, kennt kein »Publikum«, keine spezifischen Empfänger ihrer Arbeit, sondern ist - mit Recht - besessen vom Ziel der objektiven Richtigkeit, der intersubjektiven Verbindlichkeit ihrer Ergebnisse, wobei Objektivität zu ihrem Problem als Wissenschaft gehört, das allein wissenschaftstheoretisch zu bewältigen 
ist, etwa in dem oben angedeuteten Sinne, daß sich das Subjekt als Instanz gegenüber dem Verstehen von Kunst gelten läßt und sich die Fähigkeit zuspricht, dem stets das Subjekt meinenden ästhetischen Gebilde die Zunge zu lösen.

Das Publikum jedoch, dem der Musikanalytiker beim Verstehen von Musik helfen möchte, ist keine objektive Menge, keine anonyme Intersubjektivität, sondern besteht aus Menschen, Gruppen von Menschen, die der Musik mit verschiedenen, sozial- oder milieugeprägten, erziehungs-, alters- und gewohnheitsbedingten Vorkenntnissen und Erwartungshaltungen gegenüberstehen. Wo das Sprechen über Musik bewußt und gezielt (und gekonnt) auf die individuelle Konstitution, die spezifischen Verstehensprämissen solcher Gruppen sich einstellt, wird Musikanalyse zur Musikvermittlung, der Analytiker zu einem Mittelsmann, der den Musikempfänger in seiner Prädisposition zugleich als in bestimmter Weise prädisponierten Empfänger seines Sprechens über Musik ansieht und - um helfen zu können - sich in jeder Beziehung auf ihn einstellt.

Dabei ist und bleibt das spezifisch Ästhetische, das der Seele zugewandte sich Mitteilen von Sinn für die Sinne in seiner Ansprechbarkeit und in seinem Dasein jenseits aller Sprache, die Hauptsache. Das Sprechen über Musik vermittelt zwischen den Prozessen der musikalischen Sinn- und Gehaltsstiftung im Objekt, das es zu erfassen sucht, und der ästhetischen Potenz im Subjekt des Empfängers, die es $\mathrm{zu}$ fördern gilt. Immer und in jedem Augenblick solcher Vermittlung sind das empfangende Subjekt und die konkrete Musik zugleich gemeint, ist durch den Empfänger hindurch die Musik und durch die Musik hindurch der Empfänger das Ziel der Sprache.

Das bedeutet zunächst, daß bei der Vermittlung von Musik das Drumherumgehen um ihre Hauptsache, das Auslassen des spezifisch Ästhetischen, gänzlich auszuschließen ist. Bei solcher weit verbreiteten Art des Sprechens über Musik wird über sie geredet, indem man ihr aus dem Wege geht. Man berichtet über Histörchen und Anekdoten oder in der Weise des Mitteilens von Umgebungsfakten, um die Musik herumgelagertem Informations-, Wissens-, Bildungsgut, das für das spezifisch ästhetische Verstehen der Musik wenig oder gar nichts ausrichtet. Die Gruppe jener Jugendlichen z. B., deren ästhetische Potenz durch Popmusik okkupiert ist, wird von dem verselbständigten Bildungssprechen (opus soundsoviel, komponiert dann und dann, unter den und den Umständen etc.) abgestoßèn, während dem Schüler im Musikunterricht auf diese Weise das schiefe Verlangen nach abfragbarem Stoff nahegelegt und zugleich gestillt wird und der Konzertabonnent sich durch solches Reden oder Schreiben in der Meinung eingelullt fühlt, als sei es schon die Sache selbst.

Indessen kann die einer Musik vor-, neben- oder nachgelagerte Faktizität durchaus genutzt werden als Einstieg ins Sprechen über Musik, als Zugang, Aufhänger oder Anreiz, sofern von hier aus ein 
Bezug zum Ästhetischen des Gegenstandes hergestellt wird. So - um hier nur einige wenige und beliebige Beispiele $\mathrm{zu}$ geben, die zudem auf die gewissermaßen alleräußerlichsten Faktizitäten Satzüberschrift, Entstehungszeit, Opuszahl eingeschränkt sind - kann bei dem 3. Satz des Streichquartetts op. 33, Nr. 5 von Haydn ausgegangen werden von der Frage, warum dieser Satz Scherzo heißt, da hier das so benannte metrische Spiel mit Erwartungshaltungen mitten in die musikalische Hauptsache hineinführt. - Die Tatsache, daß Beethovens I. Symphonie 1799/1800 komponiert wurde, hat als bloße Nennung keinen Aussagewert für das ästhetische Verstehen der Musik, kann aber einen solchen gewinnen, indem klargemacht wird, daß dies etwa im Vergleich mit Mozart ein sehr spätes Datum ist. Von hier aus gibt es eine direkte Verbindung $\mathrm{zu}$ dem gesteigerten Selbstanspruch eines gewandelten kompositorischen Denkens und - damit zusammenhängend - zu dem völlig neuen ethischen Grundzug, der schon den 1. Satz prägt (mächtiger C-dur-Schluß - Einleitung, die das C-dur umgeht Willensimpuls des 1 . Themas; Verdeutlichung möglich durch Vergleich mit dem Anfang der Jupiter-Symphonie von Mozart). Und von hier wiederum gibt es einen Weg zu dem gesteigerten Originalitätsanspruch des Komponierens, der durch die wachsende Öffentlichkeitsgeltung (Wiederholbarkeit, höherer Gebrauchsanspruch) der Symphonik begründet werden kann. - Die Angabe »op. 2« bei Schuberts Gretchen am Spinnrade ist an sich nichtssagend, kann aber zum Sprechen gebracht werden in bezug zur Komposition des Liedes durch den Hinweis darauf, daß Schubert sein Schaffen mit den ersten Vertonungen von Goethe-Gedichten als Opera zu zählen begann (op. 1, Erlkönig), indem es die Art des Goethe-Gedichtes war, die den eigentlichen Typus des Schubert-Liedes entstehen ließ.

Die Basis der Musikvermittlung ist die Musikanalyse als Erkundung von Sinn und Gehalt der zu vermittelnden Musik unter Einsatz aller methodologischen Möglichkeiten, die die Analyse als Wissenschaft bietet. Die wissenschaftlichen Prozeduren, Erkenntnisse und Beweisführungen kommen im Akt der Musikvermittlung jedoch nicht direkt, sondern stets verwandelt zur Sprache, umgeformt und verarbeitet in die Richtung des Empfängers des Sprechens über Musik, der konkreten Zielgruppe, der die Musik in ihrer Hauptsache als ästhetisches Gebilde näher gebracht werden soll. Je intensiver die Analyse erfolgt ist, d. h. je besser (tiefgreifender) die Person des Vermittlers selbst die Musik im sprachlichen Zugriff verstanden hat, desto souveräner kann die Vermittlung erfolgen, d.h. desto freier und einfallsreicher kann sich das Sprechen auf die Zielgruppe einstellen.

Solche Zielgruppen sind z. B. die Arbeitnehmer in verschiedenen Betrieben, die Berufsmusiker (Orchestermitglieder, Opernsänger), die Kinder und Jugendlichen in den Musikschulen, die Schüler der verschiedenen Schularten und -klassen, die nach Interessenokkupation, nach Alter, städtischer und ländlicher Herkunft verschiedenen Jugend- 
lichengruppen als Konsumenten der Massenmedien, die Zielgruppen der verschiedenen Wellen und Sendezeiten des Rundfunks, das Konzertund Festivalpublikum, die Besucher von Jugendkonzerten, die erwachsenen Besucher von Volkshochschulkursen. Die Zielgruppen in ihrer gruppenspezifischen Befindlichkeit, ihren subjektiven Prädispositionen und deren Verursachungen und Bedingungen müssen von dem Vermittler der Musik ebenso studiert und verstanden worden sein, wie er die Musik, die er vermitteln will, studiert und verstanden haben muß.

Daher ist für die Musikvermittlung - anders als für die Musikanalyse - die Verbindung von Musikwissenschaft, Soziologie und Pädagogik höchst erstrebenswert und noch zu bereichern durch dasjenige, was man nicht aus Büchern lernen kann, nämlich durch die gezielte Arbeit an der Gewinnung einer die Gruppenstruktur der Gesellschaft betreffenden praktischen Gegenwartskenntnis in der Weise gleichsam persönlicher Feldforschung. Wo aber aus Mangel an Studium und Erfahrung auf die gruppenspezifische Perfektion zunächst verzichtet und an eine mehr oder weniger gemischte Liebhaber- und Laiengruppe gedacht werden muß, da wäre schon viel gewonnen, wenn nur überhaupt man nach Wegen der Vermittlung sucht, auf denen weder um die Musik herumgegangen wird oder ihre ästhetische Hauptsache dem Dilettantismus preisgegeben ist, noch die akademische Gelehrsamkeit herrscht, sondern auf denen die Musikanalyse gewissermaßen humanisiert wird, indem das Sprechen über Musik von dem Objektivitätscharakter der Wissenschaft (ohne ihn $\mathrm{zu}$ verraten) sich freimacht, zugunsten der Menschen, an die das Sprechen sich wendet.

Eine sehr wesentliche Rolle spielt dabei die Person des Musikvermittlers, seine Ausstrahlung, jenes gewisse Etwas, das nur bedingt zu erlernen ist. Dies freilich gilt für die Pädagogik überhaupt, z. B. für den Lehrer in der Schule, und darüber hinaus für jede Zwischenschaltung einer Person in einem Darbietungsakt, z. B. für den Diskjockey der Diskothek und für den Moderator in Hörfunk und Fernsehen. Aber für die Musikvermittlung gilt dies eben auch, und zusätzlich darin unterscheidet sie sich von der akademischen Musikanalyse. Während sich der Vermittler sowohl mit der Musik identifiziert, die er vermittelt, als auch mit der Gruppe, auf die sein Sprechen über Musik abzielt, ist für den Empfänger der Vermittler die primäre Identifikationsinstanz. In seinem Auftreten, seinem Tonfall, seiner Art des Sprechens und Schreibens, des Sagens und Besagens vermag ein Inneres sich kundzutun, jene Menschlichkeit, Lebenswärme und Einfühlungskraft, Erlebnisfähigkeit und -tiefe, die Vertrauen weckt, Sympathie, Identifikation und die die Hürden abzubauen hilft: die negativen Konnotationen, die mit der Opusmusik verbunden sein können, oder den Irrtum, nicht musikalisch zu sein, oder das Gefühl der Unsicherheit gegenüber Musik, oder das Fremdsein bestimmter Musikarten, oder die Opposition gegenüber dem Sprechen über Musik 
überhaupt, die Scheu vor dem Begriff, die Angst vor dem NichtVerstehen und Versagen.

Wichtig schließlich ist für die Musikvermittlung auch dasjenige, was hier der Vermittlungsansatz genannt werden kann, die Vermittlungsidee, der schöpferische Einfall. Dessen Nährboden ist der Vermittlungswille, die Intensität des Verlangens, einen Schlüssel zu finden, der die Musik aufschließt und zugleich die Seele des Empfängers. Solche Schlüssel müssen für jeden Vermittlungsakt neu gefunden werden, und hierfür gibt es keine Rezepte. Lehrbar zunächst ist jedoch das Bewußtsein für die Notwendigkeit des Suchens nach Vermittlungseinfällen, und weitgehend erlernbar sind die Voraussetzungen, die das Finden erleichtern, vorab die analytische Erkundung der Musik und das Sich-Einstellen auf die Zielgruppe, wobei dann das Sprechen, indem es durch die subjektive Betroffenheit des Vermittlers hindurchgeht oder aus ihr stammt, zugleich den musikalischen Befund, diese ästhetische Hauptsache zu erfassen und ihn in die begriffliche Unerreichbarkeit seines spezifisch ästhetischen Daseins zu öffnen versucht.

Schlüssel gibt es viele, für jede Musik eine wohl unerschöpfliche Menge. Und ganz verschiedenartig können die Ansatzpunkte sein.

Vom Erleben z. B. gehe ich aus, wenn ich im Blick auf Schuberts Lied Letzte Hoffnung über das Weinen spreche, was es für den Menschen sein kann und was es für Schubert ist, indem sein Lied bei der letzten Gedichtzeile (»wein auf meiner Hoffnung Grab») in das emphatisch Schöne des liedhaften, melismatisch schluchzenden, vorhaltsreichen Dur-Gesanges sich entlädt, der - im Kontrast zu den Abbildern des Unentschiedenen, des Zitterns und des Fallens $\rightarrow$ schon zuvor bei den Schlüssen der Doppelverse sich anbahnte.

Von einer Veranschaulichung des einen durch ein anderes gehe ich aus, wenn ich aus dem Anfang der Klaviersonate G-dur von Mozart eine Dialogszene mache, indem ich die Motive mit Text singe, auf zwei Personen verteilt, etwa: »Hast mich lieb? « — »Ja, sehr.» »Wirklich lieb? « — »Komm her.« - Beide: »Ja, wir lieben uns, lieben uns so sehr, was bedarf es denn mehr «, von wo aus unmittelbar der Satztypus Melodie und Begleitung, das periodenmetrische Prinzip des Aufstellens und Beantwortens und auch der zweitaktige Hemioleneinschub vor dem eigentlich achten Periodentakt in die Sphäre des erkennenden Verstehens von Musik erhoben werden können.

Von der Imagination, die Musik zu erzeugen vermag, gehe ich aus, wenn ich über die zweite Nachtmusik der VII. Symphonie von Mahler sage, wie ich sie höre, dieses Andante amoroso, - "gehend", »lieblich «, und es zeige und vorspiele: Mahler geht, schreitet - durch Wien, abends, nachts - und er liebt das, was es da zu hören gibt, und fängt es ein, was da an Musik erklingt auf den Straßen, den Plätzen, in den Kaffeehäusern: violinenselige Heurigenmusik, Mando- 
linengezittertes, Gitarrengespiel, Klarinettengetön, Geschrammeltes, Geklimpertes, - und dann geht er, schreitet weiter, wohlig, glücklich, und dann klingt es wieder: das Stimmen von Instrumenten, Hornruf des Wächters, Melodiegespiele und Klanggezupftes von links und rechts, auch mal so etwas aus dem Opernhaus und dem musikalischen Volkstheater, und dann geht er weiter, ganz voll von wohlig Schönem, und dann klingt es wieder, - und am Schluß machen die Kaffeehäuser dicht, wird das Gespiele immer weniger und dünner, gehen die Lichter aus, wird die Nacht still.

Die Musikvermittlung ist heute, in einer Zeit des Anwachsens eines allgemeinen Interesses an Musik und der sich noch immer verstärkenden Rolle der Massenmedien, ein zunehmendes Erfordernis und dies immer mehr auch im Rahmen beruflicher Tätigkeiten. Kann man dem Gedankengang meines Textes zustimmen, so ergibt sich, daß die Praxis der Musikvermittlung auf die Wissenschaft der Musikanalyse (und darüber hinaus auch etwa auf psychologische, sozial- und erziehungswissenschaftliche Studien) angewiesen ist und sich auf sie angewiesen wissen sollte, um von hier aus jenen festen Boden unter den Füßen $\mathrm{zu}$ gewinnen, der für die schöpferische Souveränität, die Lebendigkeit und Einfallsfreiheit des auf eine Zielgruppe gerichteten Sprechens über Musik eine wesentliche und eine weitgehend erlernbare Voraussetzung ist. Andererseits sollte an den musikwissenschaftlichen Arbeits- und Ausbildungsstätten, den Universitäten, Hochschulen und Akademien, die Musikanalyse nicht ausschließlich als Wissenschaft Geltung haben, die als solche gewissermaßen nur sich selber meint, sondern es sollte hier das Sprechen über Musik auch in Richtung der Vermittlungspraxis durchdacht, geöffnet und verwandelt und die Vermittlung von Musik als Dienst am Menschen $\mathrm{zu}$ einem Gegenstand des Forschens, Lehrens und Publizierens erhoben werden.

\section{POVZETEK}

Avtor razlaga posredovanje glasbe kot obravnavo glasbe, ki temelji na glasbeni analizi, a je vendar usmerjena na določeno skupino poslušalcev oziroma interesentov. V času, ko narašča splošno zanimanje za glasbo in se krepi vloga množičnih občil, je tudi vse bolj potrebno posredovanje glasbe in to $\mathrm{v}$ okviru poklicne dejavnosti. Iz avtorjevih izvajanj sledi, da se praksa posredovanja glasbe naslanja na znanstveno glasbeno analizo (razen tega pa še na psihološke, sociološke in pedagoške študije), na katero mora biti nujno vezana, kajti le tako si lahko ustvari trdno podlago, ki je prvi in bistveni pogoj za suvereno, živo in domiselno razlaganje glasbe, namenjene določeni skupini. Vsekakor pa glasbena analiza ne bi smela $v$ okviru muzikoloških kateder in ustanov kakor tudi visokih šol in akademij veljati izključno kot znanost. Treba bi bilo razmisliti o razlaganju glasbe iz perspektive prakse posredovanja in le-to povzdigniti $\mathrm{v}$ predmet preučevanja, učenja in publiciranja, za kar naj bi dal spodbudo tudi pričujoči prispevek. 